AS-ITP-95-16

April 29, 1995

\title{
Three-Dimensional Vertex Model Related BCC Model in Statistical Mechanics
}

\author{
Zhan-Ning Hu 由 \\ CCAST(WORLD LABORATORY) P.O.BOX 8730, BEIJING, 100080 \\ and \\ INSTITUTE OF THEORETICAL PHYSICS, ACADEMIA SINICA, \\ P. O. BOX 2735 BEIJING 100080, CHINA \&
}

\begin{abstract}
In this paper, a three-dimensional vertex model is obtained. It is a duality of the three-dimensional integrable lattice model with $N$ states proposed by Boos, Mangazeev, Sergeev and Stroganov. The Boltzmann weight of the model is dependent on four spin variables, which are the linear combinations of the spins on the corner sites of the cube, and obeys the modified vertex type tetrahedron equation. This vertex model can be regard as a deformation of the one related the threedimensional Baxter-Bazhanov model. The constrained conditions of the spectrums are discussed in detail and the symmetry properties of weight functions of the vertex model are presented.
\end{abstract}

Keywords: Three-dimensional vertex model; Boltzmann weights; Modified vertex type tetrahedron equation; Body-Centered-Cube model; Constrained conditions.

\footnotetext{
${ }^{1}$ email address: huzn@itp.ac.cn

${ }^{2}$ mail address
} 


\section{Introduction}

There is a large class of integrable lattice models in statistical mechanics. Most of them are two-dimensional, such as Ising model [1], six-vertex model [2], eightvertex model [3], chiral Potts model 44] et al, where the Yang-Baxter relation (or the star-triangle relation) plays an important role. It ensures the commutativity of the transfer-matrices, then permits us to calculate the partition function per site in the thermodynamical limit. Recently much attention has been payed on the three-dimensional integrable lattice model. Bazhanov and Baxter generalized the Zamolodchikov's two states model [5] to the arbitrary states [6] and Kashaev et al showed that the tetrahedron equation holds in this model by introducing the starsquare relation [7, 8, 9, 10]. The symmetry properties of the weight functions have been discussed in ref [11, 12, 13]. Boos and Mangazeev et al obtained another kind of the lattice model in three-dimensions.

One of the other approaches of the three-dimensional integrable models is of the vertex typed models. Hietarinta discussed the tetrahedron equation by considering the scattering of straight strings [14], similarly as in ref [5], where the three labeling schemes exist. The duality between them is discussed in detail. Then the main problems are to solved these tetrahedron equations and to build the integrable models. In fact, Korepanov [15] given the solutions of the vertex type tetrahedron equation for the two states in 1993, which is different from the ones in [14]. The vertex type tetrahedron equations are discussed also in refs. [16, 17, 18]. Another kind of the vertex model in three dimensions is proposed in ref. [19]. Now we know that the Boltzmann weight of this model can be obtained from that of the Baxter-Bazhanov model [20]. And the three-dimensional vertex model as the correspondence of Baxter-Bazhanov model has been built in ref. [21]. In this paper, we obtained the three-dimensional vertex model corresponding to the lattice integrable model proposed in ref. [22, 23]. It can be regard as a deformation of the three-dimensional vertex model in [21]. The connections of the restricted conditions of the spectrums between these models are discussed in detail and the symmetry 
properties are presented also. The weight function of the model depends on the four spins variables and satisfies the modified vertex type tetrahedron equation.

The organization of this paper is as follows. In section 2, some notations and the necessary definitions are given and the weight functions of the lattice integrable model are presented. They satisfy the modified tetrahedron equations. In section 3, we find that the weight function of the model is dependent on four spins variables which are the linear combinations of the spins located on the corner sites of the cubes. We formulate the weight functions as the vertex forms. They satisfy the modified vertex type tetrahedron equations. In section 4 we compare the weight functions between this model and the three-dimensional vertex model related the Baxter-Bazhanov model. The former can be regard as a deformation of the later. Then the connections of the restricted conditions for the two kinds of vertex models are discussed in detail. In section 5, the symmetry properties are presented, which are similar as in ref. [21]. As the short conclusions, some remarks are given finally.

\section{The Body-Centered-Cube Model}

In this section, the brief describe of the lattice integrable model proposed in [22, 223 is given and the necessary definitions and some notations are presented. The weight functions of this model have the Body-Centered-Cube form, similarly as the BaxterBazhanov model, and satisfy the modified tetrahedron equation which leads to the commuting family of two-layer transfer-matrices. The simple cubic lattice $\mathcal{L}$ is consisted of two types of elementary cubes alternating in checkerboard order in all directions and at each site of $\mathcal{L}$ place a spin with $Z_{N}$ values for integer $N \geq 2$.

The weight function $W(a|e f g| b c d \mid h)$ of Body-Center-Cube (BCC) model (see ref. [22]) depends on the eight surrounding spin variables $a, b, \cdots, h$ and has the form

$$
\begin{aligned}
& W_{P}(a|e f g| b c d \mid h) \\
& \quad=\left[\frac{w\left(x_{58} x_{67}, x_{8} \mu, x_{13} x_{24} x_{7} x_{8} / x_{3} x_{4} \mid a+d, e+f\right)}{w\left(x_{58} x_{67}, x_{5} \nu, x_{13} x_{24} x_{5} x_{6} / x_{1} x_{2} \mid g+h, c+b\right)}\right]^{1 / 2}
\end{aligned}
$$




$$
\begin{aligned}
& \times\left[\frac{w\left(x_{1} x_{8}, x_{1} u, x_{3} x_{5} \mid e+h, d+c\right)}{w\left(x_{4} x_{6}, x_{2} v, x_{2} x_{7} \mid a+b, f+g\right)}\right]^{1 / 2} \\
& \times\left[\frac{w\left(x_{2} x_{8}, x_{4} \lambda, x_{4} x_{5} \mid e+g, a+c\right)}{w\left(x_{3} x_{6}, x_{3} \xi, x_{1} x_{7} \mid b+d, f+h\right)}\right]^{1 / 2} \frac{\omega^{b f} \omega^{(a g+g b+b h) / 2}}{\omega^{a g} \omega^{(h d+d e+e a) / 2}} \\
& \quad \times\left\{\sum_{\sigma \in Z_{N}} \frac{w\left(x_{3}, x_{13}, x_{1} \mid d, h+\sigma\right) w\left(x_{4}, x_{24}, x_{2} \mid a, g+\sigma\right)}{w\left(x_{8}, x_{58}, x_{5} \mid e, c+\sigma\right) w\left(x_{7} / \omega, x_{67}, x_{6} \mid f, b+\sigma\right)}\right\}_{0}
\end{aligned}
$$

where the subscript " 0 " after the curly brackets indicates that the expression in the braces is divided by itself with the zero exterior spins. The function $w(x, y, z \mid k, l)$ is defined as

$$
w(x, y, z \mid k, l)=w(x, y, z \mid k-l) \Phi(l), \quad w(x, y, z \mid l)=\prod_{j=1}^{l} \frac{y}{z-x \omega^{j}}, \quad k, l \in Z_{N}
$$

with the notation

$$
x^{N}+y^{N}=z^{N}, \quad \Phi(l)=\omega^{l(l+N) / 2}, \quad \omega^{1 / 2}=\exp (\pi i / N) .
$$

The relation $x^{N}+y^{N}=z^{N}$ decides the variables $x_{13}, x_{24}, x_{58}, x_{67}$ and $u, v, \xi, \lambda, \mu, \nu$ up to some $\omega$ factors. The weight function satisfies the modified tetrahedron equation 24

$$
\begin{aligned}
\sum_{d} W\left(a_{4}\left|c_{2} c_{1} c_{3}\right| b_{1} b_{3} b_{2} \mid d\right) \bar{W}^{\prime}\left(c_{1}\left|b_{2} a_{3} b_{1}\right| c_{4} d c_{6} \mid b_{4}\right) \\
\times W^{\prime \prime}\left(b_{1}\left|d c_{4} c_{3}\right| a_{2} b_{3} b_{4} \mid c_{5}\right) \bar{W}^{\prime \prime \prime}\left(d\left|b_{2} b_{4} b_{3}\right| c_{5} c_{2} c_{6} \mid a_{1}\right) \\
=\sum_{d} W^{\prime \prime \prime}\left(b_{1}\left|c_{1} c_{4} c_{3}\right| a_{2} a_{4} a_{3} \mid d\right) \bar{W}^{\prime \prime}\left(c_{1}\left|b_{2} a_{3} a_{4}\right| d c_{2} c_{6} \mid a_{1}\right) \\
\times W^{\prime}\left(a_{4}\left|c_{2} d c_{3}\right| a_{2} b_{3} a_{1} \mid c_{5}\right) \bar{W}\left(d\left|a_{1} a_{3} a_{2}\right| c_{4} c_{5} c_{6} \mid b_{4}\right)
\end{aligned}
$$

where $W, W^{\prime}, W^{\prime \prime}, W^{\prime \prime \prime}$ and $\bar{W}, \bar{W}^{\prime}, \bar{W}^{\prime \prime}, \bar{W}^{\prime \prime \prime}$ are four independent pairs of weight functions. It reduces to Baxter-Bazhanov model when $W=\bar{W}$. The function $w(x, y, z \mid l)$ has the property

$$
w(x, y, z \mid l) w\left(z, \omega^{1 / 2} y, \omega x \mid-l\right) \Phi(l)=1, \quad l \in Z_{N} .
$$

We will formulate the weight functions of this model as the vertex form in the following section and find that the function $W($ a $\mid$ efg $g|b c d| h)$ is dependent on four spin variables which are the linear combinations of the spins $a, b, \cdots, h$. 


\section{Three-Dimensional Vertex Model}

In this section the three-dimensional vertex model is obtained. It is a duality of the 3D integrable lattice model presented above and can be regarded as a deformation of the vertex model given in ref. [21].

Set

$$
k_{1}=f+h-b-d, \quad k_{2}=b+c-g-h, \quad k_{3}=e+h-c-d, \quad k_{4}=a+h-d-g .
$$

By taking account of the relation (5), we can express the weight function (1) as:

$$
\begin{gathered}
W(a|e f g| b c d \mid h)=(-)^{k_{2}}\left(\omega^{1 / 2}\right)^{k_{1} k_{2}+k_{2} k_{3}+k_{1} k_{3}}\left[\frac{w\left(x_{1} x_{7}, \omega^{1 / 2} x_{3} \xi, \omega x_{3} x_{6} \mid k_{1}\right)}{w\left(x_{4} x_{5}, \omega^{1 / 2} x_{4} \lambda, \omega x_{2} x_{8} \mid k_{4}-k_{3}\right)}\right. \\
\left.\times \frac{w\left(x_{58} x_{67}, x_{8} \mu, x_{13} x_{24} x_{7} x_{8} / x_{3} x_{4} \mid k_{4}-k_{1}-k_{2}-k_{3}\right) w\left(x_{1} x_{8}, x_{1} u, x_{3} x_{5} \mid k_{3}\right)}{w\left(x_{58} x_{67}, x_{5} \nu, x_{13} x_{24} x_{5} x_{6} / x_{1} x_{2} \mid-k_{2}\right) w\left(x_{4} x_{6}, x_{2} \nu, x_{2} x_{7} \mid k_{4}-k_{1}\right)}\right]^{1 / 2} \\
\times\left\{\sum_{\sigma \in Z_{N}} \frac{\omega^{\sigma k_{2}} w\left(x_{3}, x_{13}, x_{1} \mid \sigma\right) w\left(x_{4}, x_{24}, x_{2} \mid k_{4}+\sigma\right)}{w\left(x_{8}, x_{58}, x_{5} \mid k_{3}+\sigma\right) w\left(x_{7} / \omega, x_{67}, x_{6} \mid k_{1}+\sigma\right)}\right\}_{0} .
\end{gathered}
$$

It is obvious that the weight function $W(a|e f g| b c d \mid h)$ depends on the spin variables $k_{1}, k_{2}, k_{3}, k_{4}$ defined in relation (6). Using the relation

$$
\begin{aligned}
\left\{\sum_{\sigma \in Z_{N}}\right. & \left.\frac{w\left(x_{3}, x_{13}, x_{1} \mid \sigma\right) w\left(x_{4}, x_{24}, x_{2} \mid \sigma+k_{4}\right) \omega^{\sigma k_{2}}}{w\left(x_{8}, x_{58}, x_{5} \mid \sigma+k_{3}\right) w\left(x_{7} / \omega, x_{67}, x_{6} \mid \sigma+k_{1}\right)}\right\}_{0} \\
= & \frac{w\left(x_{4} x_{6}, x_{2} v, x_{2} x_{7} \mid k_{4}-k_{1}\right)}{w\left(x_{1} x_{8}, x_{1} u, x_{3} x_{5} \mid k_{3}\right)} \\
& \times\left\{\sum_{\sigma \in Z_{N}} \frac{w\left(x_{8} x_{13}, x_{1} u, x_{3} x_{58} \mid \sigma+k_{3}+k_{2}\right) w\left(x_{2} x_{67}, x_{2} v, x_{6} x_{24} \mid \sigma\right) \omega^{\sigma k_{1}}}{w\left(x_{13}, \omega x_{1} u, \omega x_{1} x_{58} \mid \sigma+k_{2}\right) w\left(x_{4} x_{67}, x_{2} v, x_{7} x_{24} \mid \sigma+k_{4}-k_{1}\right)}\right\}_{0}
\end{aligned}
$$

and fixing the normalizations of all parameters $x^{\prime} s$ appeared in the expression (1) as (see ref.22])

$$
x_{3}=x_{4}=x_{7}=x_{8}=1,
$$

we can change the weight function (7) into the vertex form

$$
R_{i_{1} i_{2} i_{3}}^{j_{1} j_{2} j_{3}}=(-)^{j_{2}}\left(\omega^{1 / 2}\right)^{j_{1} j_{2}+j_{2} j_{3}+j_{1} j_{3}}
$$




$$
\begin{gathered}
\times\left[\frac{w\left(x_{1}, \omega^{1 / 2} \xi, \omega x_{6} \mid j_{1}\right) w\left(x_{58} x_{67}, \mu, x_{13} x_{24} \mid-i_{2}\right) w\left(x_{6}, x_{2} v, x_{2} \mid i_{3}\right)}{w\left(x_{5}, \omega^{1 / 2} \lambda, \omega x_{2} \mid i_{1}\right) w\left(x_{58} x_{67}, x_{5} \nu, x_{13} x_{24} x_{5} x_{6} / x_{1} x_{2} \mid-j_{2}\right) w\left(x_{1}, x_{1} u, x_{5} \mid j_{3}\right)}\right]^{1 / 2} \\
\times\left\{\sum_{\sigma \in Z_{N}} \frac{w\left(x_{13}, x_{1} u, x_{58} \mid \sigma+j_{2}+j_{3}\right) w\left(x_{2} x_{67}, x_{2} v, x_{6} x_{24} \mid \sigma\right) s\left(\sigma, j_{1}\right)}{w\left(x_{5} x_{13}, \omega x_{1} u, \omega x_{1} x_{58} \mid \sigma+j_{2}\right) w\left(x_{67}, x_{2} v, x_{24} \mid \sigma+i_{3}\right)}\right\}_{0}
\end{gathered}
$$

where

$$
\begin{array}{ll}
i_{1}=k_{4}-k_{3}, & j_{1}=k_{1}, \\
i_{2}=k_{1}+k_{2}+k_{3}-k_{4}, & j_{2}=k_{2}, \\
i_{3}=k_{4}-k_{1}, & j_{3}=k_{3} .
\end{array}
$$

Set

$$
\frac{x_{5}}{\omega x_{2}}=q^{-1} u_{1}, \quad \frac{u_{2}^{\prime}}{u_{2}^{\prime \prime}}=q u_{2}, \quad \frac{x_{2} x_{13}}{\omega x_{6} x_{58}}=q u_{2}^{\prime}, \quad \frac{x_{2} x_{67}}{x_{6} x_{24}}=q u_{2}^{\prime \prime}, \quad \frac{x_{6}}{x_{2}}=q^{-1} u_{3}
$$

where

$$
x_{1} x_{2}=q^{2} x_{5} x_{6}
$$

By considering the notation [6]

$$
\frac{w(v, a)}{w(v, 0)}=[\Delta(v)]^{a} \prod_{j=1}^{a}\left(1-\omega^{j} v\right)^{-1}, \quad \Delta(v)=\left(1-v^{N}\right)^{1 / N},
$$

the weight function has the form

$$
\begin{aligned}
R\left(u_{1}, u_{2}, u_{3}\right)_{i_{1} i_{2} i_{3}}^{j_{1} j_{2} j_{3}}=(-)^{j_{2}}\left(\omega^{1 / 2}\right)^{j_{1} j_{2}+j_{2} j_{3}+j_{1} j_{3}} & \\
& \times\left[\frac{w\left(q u_{1}, j_{1}\right) w\left(q^{-1}\left(\omega u_{2}\right)^{-1},-i_{2}\right) w\left(q^{-1} u_{3}, i_{3}\right)}{w\left(q^{-1} u_{1}, i_{1}\right) w\left(q\left(\omega u_{2}\right)^{-1},-j_{2}\right) w\left(q u_{3}, j_{3}\right)}\right]^{1 / 2} \\
& \times\left\{\sum_{\sigma \in Z_{N}} \frac{w\left(\omega u_{2}^{\prime} u_{3}, \sigma+j_{2}+j_{3}\right) w\left(q u_{2}^{\prime \prime}, \sigma\right) s\left(\sigma, j_{1}\right)}{w\left(q^{-1} u_{2}^{\prime}, \sigma+j_{2}\right) w\left(u_{2}^{\prime \prime} u_{3}, \sigma+i_{3}\right)}\right\}_{0} .
\end{aligned}
$$

Similarly as in ref. [22], we can express the spectrums $u_{i}(i=1,2,3)$ as

$$
u_{i}^{N}=\frac{C_{i}+D_{i}}{C_{i}-D_{i}}
$$

where $C_{i}, D_{i}$ satisfy the relation

$$
\frac{1-D_{i}^{2}}{1-C_{i}^{2}}=\frac{\left(1-q^{N}\right)^{2}}{\left(1+q^{N}\right)^{2}}, \quad i=1,2,3 .
$$


Then let

$$
\cos \left(a_{0}\right)=\frac{k^{2} C_{1} C_{2} C_{3}-D_{1} D_{2} D_{3}}{k^{\prime 2}}, \quad \cos \left(a_{r}\right)=\frac{(-)^{r}\left(D_{p} D_{q} C_{r}-C_{p} C_{q} D_{r}\right)}{k^{\prime 2} S_{p} S_{q}},
$$

where $\{p, q, r\}=\{1,2,3\}$ with

$$
C_{i}^{2}+S_{i}^{2}=1, \quad k^{2}+k^{2}=1, \quad k=\frac{1-q^{N}}{1+q^{N}}
$$

We can denote the variables $x_{i}^{N}$ as

$$
\begin{aligned}
& x_{1}^{N}=-\left(u_{1} u_{3}\right)^{N / 2} \sqrt{\frac{1-\left(q u_{2}\right)^{N}}{1-\left(q^{-1} u_{2}\right)^{N}}} \exp \left(i a_{2}\right), \\
& x_{2}^{N}=-\left(u_{1} u_{3}\right)^{-N / 2} \sqrt{\frac{1-\left(q u_{2}\right)^{N}}{1-\left(q^{-1} u_{2}\right)^{N}}} \exp \left(i a_{2}\right), \\
& x_{5}^{N}=-q^{-N}\left(u_{1} u_{3}^{-1}\right)^{N / 2} \sqrt{\frac{1-\left(q u_{2}\right)^{N}}{1-\left(q^{-1} u_{2}\right)^{N}}} \exp \left(i a_{2}\right), \\
& x_{6}^{N}=-q^{-N}\left(u_{1}^{-1} u_{3}\right)^{N / 2} \sqrt{\frac{1-\left(q u_{2}\right)^{N}}{1-\left(q^{-1} u_{2}\right)^{N}}} \exp \left(i a_{2}\right),
\end{aligned}
$$

where the phases are chosen by

$$
\frac{x_{1}}{\left|x_{1}\right|}=\frac{x_{2}}{\left|x_{2}\right|}=\frac{x_{5}}{\omega^{1 / 2}\left|x_{5}\right|}=\frac{\omega^{1 / 2} x_{6}}{\left|x_{6}\right|}=e^{i a_{2} / N} .
$$

And the expressions of the variables $x_{13}, x_{24}, x_{58}, x_{67}$ can be obtained by

$$
x_{13}^{N}=x_{1}^{N}-1, \quad x_{24}^{N}=x_{2}^{N}-1, \quad x_{58}^{N}=x_{5}^{N}-1, \quad x_{67}^{N}=x_{6}^{N}-1
$$

from relations (20) with the phases

$$
\begin{array}{lll}
\frac{x_{13}}{\left|x_{13}\right|}=e^{i\left(a_{0}+a_{1}+a_{2}+a_{3}\right) /(2 N)}, & \frac{x_{24}}{\left|x_{24}\right|}=\omega^{1 / 2} e^{i\left(a_{0}-a_{1}+a_{2}-a_{3}\right) /(2 N)}, \\
\frac{x_{58}}{\left|x_{58}\right|}=\omega^{1 / 2} e^{i\left(a_{0}-a_{1}+a_{2}+a_{3}\right) /(2 N)}, & \frac{x_{67}}{\left|x_{67}\right|}=\omega^{-1 / 2} e^{i\left(a_{0}+a_{1}+a_{2}-a_{3}\right) /(2 N) .} .
\end{array}
$$

In this way, the weight function (15) satisfies the modified vertex type tetrahedron equation

$$
\begin{aligned}
& \sum_{\substack{\left\{k_{i}\right\}, i=1, \cdots, 6}} R\left(u_{1}, u_{2}, u_{3}\right)_{i_{1}, i_{2}, i_{3}}^{k_{1}, k_{2}, k_{3}} \bar{R}\left(u_{1}, u_{4}, u_{5}\right)_{k_{1} i_{4} i_{5}}^{j_{1} k_{4} k_{5}} R\left(u_{2}, u_{4}, u_{6}\right)_{k_{2} k_{4} i_{6}}^{j_{2} j_{4} k_{6}} \bar{R}\left(u_{3}, u_{5}, u_{6}\right)_{k_{3} k_{5} k_{6}}^{j_{3} j_{5} j_{6}}= \\
& \quad \sum_{\substack{\left\{k_{i}\right\}, i=1, \cdots, 6}} R\left(u_{3}, u_{5}, u_{6}\right)_{i_{3}, i_{5}, i_{6}}^{k_{3}, k_{5}, k_{6}} \bar{R}\left(u_{2}, u_{4}, u_{6}\right)_{i_{2} i_{4} k_{6}}^{k_{2} k_{4} j_{6}} R\left(u_{1}, u_{4}, u_{5}\right)_{i_{1} k_{4} k_{5}}^{k_{1} j_{4} j_{5}} \bar{R}\left(u_{1}, u_{2}, u_{3}\right)_{k_{1} k_{2} k_{3}}^{j_{1} j_{2} j_{3}}
\end{aligned}
$$


where $\bar{R}\left(u_{1}, u_{2}, u_{3}\right)$ can be obtained from $R\left(u_{1}, u_{2}, u_{3}\right)$ by the substitutions:

$$
q \rightarrow q^{-1}, \quad u_{2}^{\prime} \rightarrow \bar{u}_{2}^{\prime}, \quad u_{2}^{\prime \prime} \rightarrow \bar{u}_{2}^{\prime \prime}
$$

and

$$
\begin{aligned}
& u_{1}=\frac{q x_{5}}{\omega x_{2}}=\frac{q^{-1} \bar{x}_{5}}{\omega \bar{x}_{2}}=\frac{q x_{5}^{\prime}}{\omega x_{2}^{\prime}}=\frac{q^{-1} \bar{x}_{5}^{\prime}}{\omega \bar{x}_{2}^{\prime}}, \\
& u_{2}=\frac{q^{-1} x_{13} x_{24}}{\omega x_{58} x_{67}}=\frac{q \bar{x}_{13} \bar{x}_{24}}{\omega \bar{x}_{58} \bar{x}_{67}}=\frac{q x_{5}^{\prime \prime}}{\omega x_{2}^{\prime \prime}}=\frac{q^{-1} \bar{x}_{5}^{\prime \prime}}{\omega \bar{x}_{2}^{\prime \prime}}, \\
& u_{3}=\frac{q x_{6}}{x_{2}}=\frac{q^{-1} \bar{x}_{6}}{\bar{x}_{2}}=\frac{q x_{5}^{\prime \prime \prime}}{\omega x_{2}^{\prime \prime \prime}}=\frac{q^{-1} \bar{x}_{5}^{\prime \prime \prime}}{\omega \bar{x}_{2}^{\prime \prime \prime}} \\
& u_{4}=\frac{q^{-1} x_{13}^{\prime} x_{24}^{\prime}}{\omega x_{58}^{\prime} x_{67}^{\prime}}=\frac{q \bar{x}_{13}^{\prime} \bar{x}_{24}^{\prime}}{\omega \bar{x}_{58}^{\prime} \bar{x}_{67}^{\prime}}=\frac{q^{-1} x_{13}^{\prime \prime} x_{24}^{\prime \prime}}{\omega x_{58}^{\prime \prime} x_{67}^{\prime \prime}}=\frac{q \bar{x}_{13}^{\prime \prime} \bar{x}_{24}^{\prime \prime}}{\omega \bar{x}_{58}^{\prime \prime} \bar{x}_{67}^{\prime \prime}} \\
& u_{5}=\frac{q x_{6}^{\prime}}{x_{2}^{\prime}}=\frac{q^{-1} \bar{x}_{6}^{\prime}}{\bar{x}_{2}^{\prime}}=\frac{q^{-1} x_{13}^{\prime \prime \prime} x_{24}^{\prime \prime \prime}}{\omega x_{58}^{\prime \prime \prime} x_{67}^{\prime \prime \prime}}=\frac{q \bar{x}_{13}^{\prime \prime \prime} \bar{x}_{24}^{\prime \prime \prime}}{\omega \bar{x}_{58}^{\prime \prime \prime} \bar{x}_{67}^{\prime \prime \prime}}, \\
& u_{6}=\frac{q x_{6}^{\prime \prime}}{x_{2}^{\prime \prime}}=\frac{q^{-1} \bar{x}_{6}^{\prime \prime}}{\bar{x}_{2}^{\prime \prime}}=\frac{q x_{6}^{\prime \prime \prime}}{x_{2}^{\prime \prime \prime}}=\frac{q^{-1} \bar{x}_{6}^{\prime \prime \prime}}{\bar{x}_{2}^{\prime \prime \prime}} .
\end{aligned}
$$

Notice that the spectrums should satisfy the conditions:

$$
\begin{aligned}
& a_{0}+a_{1}^{\prime}-a_{1}^{\prime \prime}+a_{1}^{\prime \prime \prime}=a_{1}+a_{0}^{\prime}-a_{2}^{\prime \prime}+a_{2}^{\prime \prime \prime}=0, \\
& a_{2}-a_{2}^{\prime}+a_{0}^{\prime \prime}+a_{3}^{\prime \prime \prime}=a_{3}-a_{3}^{\prime}+a_{3}^{\prime \prime}+a_{0}^{\prime \prime \prime}=0,
\end{aligned}
$$

where $a_{i}^{\prime}, a_{i}^{\prime \prime}, a_{i}^{\prime \prime \prime}(i=0,1,2,3)$ are defined similarly as in relations (16) and (18) (see ref. 22]). So we get a three-dimensional vertex model which is a duality of the BCC model. It is a deformation of the three-dimensional vertex model in ref. [21]. The details will be given in the following section. And the constrained conditions of the spectrums between them are discussed.

\section{The Additional Constraints of the Parameters}

By setting $q=1$, the weight function (15) becomes as

$$
\begin{aligned}
R\left(u_{1}, u_{2}, u_{3}\right)_{i_{1} i_{2} i_{3}}^{j_{1} j_{2} j_{3}} & =(-)^{j_{2}}\left(\omega^{1 / 2}\right)^{j_{1} j_{2}+j_{2} j_{3}+j_{1} j_{3}} \\
\times & {\left[\frac{w\left(u_{1}, j_{1}\right) w\left(\left(\omega u_{2}\right)^{-1},-i_{2}\right) w\left(u_{3}, i_{3}\right)}{w\left(u_{1}, i_{1}\right) w\left(\left(\omega u_{2}\right)^{-1},-j_{2}\right) w\left(u_{3}, j_{3}\right)}\right]^{1 / 2} }
\end{aligned}
$$




$$
\times\left\{\sum_{\sigma \in Z_{N}} \frac{w\left(\omega u_{2}^{\prime} u_{3}, \sigma+j_{2}+j_{3}\right) w\left(u_{2}^{\prime \prime}, \sigma\right) s\left(\sigma, j_{1}\right)}{w\left(u_{2}^{\prime}, \sigma+j_{2}\right) w\left(u_{2}^{\prime \prime} u_{3}, \sigma+i_{3}\right)}\right\}_{0} .
$$

It is justly the weight function of the $3 \mathrm{D}$ vertex model [21] related Baxter-Bazhanov model. In this case the modified vertex type tetrahedron equation (23) reduces to the ordinary one. We know that the four additional constraints are

$$
\begin{gathered}
\omega \frac{x_{23}}{x_{3}} \frac{x_{4}^{\prime}}{x_{24}^{\prime}} \frac{x_{24}^{\prime \prime}}{x_{2}^{\prime \prime}} \frac{x_{2}^{\prime \prime \prime}}{x_{24}^{\prime \prime \prime}}=1, \quad \frac{x_{13}}{x_{1}} \frac{x_{1}^{\prime}}{x_{14}^{\prime}} \frac{x_{14}^{\prime \prime}}{x_{1}^{\prime \prime}} \frac{x_{1}^{\prime \prime \prime}}{x_{14}^{\prime \prime \prime}}=1, \\
\frac{x_{14}}{x_{4}} \frac{x_{4}^{\prime}}{x_{14}^{\prime}} \frac{x_{14}^{\prime \prime}}{x_{4}^{\prime \prime}} \frac{x_{4}^{\prime \prime \prime}}{x_{24}^{\prime \prime \prime}}=1, \quad \frac{x_{13}}{x_{3}} \frac{x_{3}^{\prime}}{x_{13}^{\prime}} \frac{x_{13}^{\prime \prime}}{x_{1}^{\prime \prime}} \frac{x_{2}^{\prime \prime \prime}}{x_{23}^{\prime \prime \prime}}=1,
\end{gathered}
$$

for the case of $q=1$. By making the transformations:

$$
\frac{x_{1}}{x_{2}} \rightarrow \frac{x_{1}}{x_{6}}, \frac{x_{1}}{x_{3}} \rightarrow x_{1}, \frac{x_{4}}{x_{3}} \rightarrow \frac{x_{1}}{x_{5}}
$$

and choosing that

$$
\frac{x_{23}}{x_{13}} \rightarrow \frac{x_{67}}{x_{13}}, \quad \frac{x_{13}}{x_{1}} \rightarrow \frac{x_{13}}{x_{1}}, \quad \frac{x_{13}}{x_{14}} \rightarrow \frac{x_{5} x_{13}}{x_{1} x_{58}}, \frac{x_{24}}{x_{23}} \rightarrow \frac{x_{1} x_{24}}{x_{5} x_{67}}
$$

we have

$$
\begin{gathered}
\frac{x_{13}}{x_{3}} \rightarrow x_{13}, \frac{x_{14}}{x_{1}} \rightarrow \frac{x_{58}}{x_{5}}, \frac{x_{23}}{x_{2}} \rightarrow \frac{x_{67}}{x_{6}}, \frac{x_{23}}{x_{3}} \rightarrow x_{67}, \\
\frac{x_{14}}{x_{4}} \rightarrow x_{58}, \quad \frac{x_{24}}{x_{2}} \rightarrow \frac{x_{24}}{x_{2}}, \frac{x_{24}}{x_{4}} \rightarrow x_{24} .
\end{gathered}
$$

Using the transformations (29), (30) and (31), we can change (28) into the form:

$$
\begin{aligned}
\omega \frac{x_{67}}{x_{24}^{\prime}} \frac{x_{24}^{\prime \prime}}{x_{2}^{\prime \prime}} \frac{x_{2}^{\prime \prime \prime}}{x_{24}^{\prime \prime \prime}} & =1, \quad \frac{x_{13}}{x_{1}} \frac{x_{5}^{\prime}}{x_{58}^{\prime}} \frac{x_{58}^{\prime \prime}}{x_{5}^{\prime \prime}} \frac{x_{5}^{\prime \prime \prime}}{x_{58}^{\prime \prime \prime}}=1, \\
\frac{x_{58}}{x_{58}^{\prime}} \frac{x_{58}^{\prime \prime}}{x_{24}^{\prime \prime \prime}} & =1, \quad \frac{x_{13}}{x_{13}^{\prime}} \frac{x_{13}^{\prime \prime}}{x_{1}^{\prime \prime}} \frac{x_{6}^{\prime \prime \prime}}{x_{67}^{\prime \prime \prime}}=1 .
\end{aligned}
$$

By comparing with the constraints for the modified tetrahedron equation introduced in ref. [22],

$$
\begin{array}{ll}
\omega \frac{x_{67}}{\bar{x}_{24}^{\prime}} \frac{x_{24}^{\prime \prime}}{x_{2}^{\prime \prime}} \frac{x_{2}^{\prime \prime \prime}}{x_{24}^{\prime \prime \prime}}=1, & \frac{\bar{x}_{13}}{\bar{x}_{1}} \frac{\bar{x}_{1}^{\prime}}{\bar{x}_{13}^{\prime}} \frac{\bar{x}_{13}^{\prime \prime}}{\bar{x}_{1}^{\prime \prime}} \frac{\bar{x}_{1}^{\prime \prime \prime}}{\bar{x}_{13}^{\prime \prime \prime}}=1, \\
\frac{x_{58}}{x_{58}^{\prime}} \frac{x_{58}^{\prime \prime}}{\bar{x}_{24}^{\prime \prime \prime}}=1, \quad \omega \frac{x_{67}^{\prime}}{\bar{x}_{24}} \frac{x_{67}^{\prime \prime \prime}}{x_{67}^{\prime \prime}}=1, \quad \frac{x_{24}}{x_{24}^{\prime}} \frac{x_{24}^{\prime \prime}}{x_{24}^{\prime \prime \prime}}=1,
\end{array}
$$

we get that the two relations in the left hand sides of (32) are corresponding to the left ones in (33) with $q=1$. It can be checked easily that the two relations in the 
right hand sides of (32) hold also by using relations (20), (21) and (22) for the case of $q=1$. And the other three relations in (33) become as

$$
\frac{x_{13}}{x_{1}} \frac{x_{1}^{\prime}}{x_{13}^{\prime}} \frac{x_{13}^{\prime \prime}}{x_{1}^{\prime \prime}} \frac{x_{1}^{\prime \prime \prime}}{x_{13}^{\prime \prime \prime}}=1, \quad \omega \frac{x_{4}}{x_{24}} \frac{x_{23}^{\prime}}{x_{3}^{\prime}} \frac{x_{3}^{\prime \prime}}{x_{23}^{\prime \prime}} \frac{x_{23}^{\prime \prime \prime}}{x_{3}^{\prime \prime \prime}}=1, \quad \frac{x_{24}}{x_{4}} \frac{x_{4}^{\prime}}{x_{24}^{\prime}} \frac{x_{24}^{\prime \prime}}{x_{4}^{\prime \prime}} \frac{x_{4}^{\prime \prime \prime}}{x_{24}^{\prime \prime \prime}}=1,
$$

by considering the variables substitutions (29), (30) and (31). These relations are correct indeed for Baxter-Bazhanov model and they are equivalent to the relation (36) of ref. [21]. The spectrums in the modified vertex type tetrahedron equation (23) can be parameterized as

$$
u_{i}=\omega^{-1 / 2}\left[\operatorname{tn}\left(\theta_{i} / 2, k\right)\right]^{-2 / N}, \quad i=1,2, \cdots, 6,
$$

where

$$
\operatorname{tn}\left(\frac{\theta_{i}}{2}, k\right)=\frac{k^{\prime} s n\left(\theta_{i} / 2, k\right)}{\operatorname{cn}\left(\theta_{i} / 2, k\right) d n\left(\theta_{i} / 2, k\right)} .
$$

When $q=1$, that is, $k=0$, they become as

$$
u_{i}=\omega^{-1 / 2}\left[\operatorname{ctg}\left(\frac{\theta_{i}}{2}\right)\right]^{2 / N}, \quad i=1,2, \cdots, 6 .
$$

And the model reduces to the 3D vertex model presented in ref. [21].

\section{$5 \quad$ Symmetry Properties and Some Remarks}

Let us denote the two generating elements of the group $G$ consisting of various rotations, reflections and their combinations of the cube as $\tau$ and $\rho$ [23, 21]. Under these transformations the parameters of the weight function change as (see ref.[22])

$$
\frac{x_{5}}{\omega x_{2}} \stackrel{\tau}{\longleftrightarrow} \frac{x_{6}}{x_{2}}, \frac{x_{13} x_{24}}{x_{58} x_{67}} \stackrel{\tau}{\longleftrightarrow} \frac{x_{13} x_{24}}{x_{58} x_{67}}
$$

and

$$
\begin{gathered}
\frac{x_{1}}{x_{5}} \stackrel{\rho}{\longleftrightarrow} \frac{x_{5}}{\omega x_{1}}, \frac{x_{2}}{x_{6}} \stackrel{\rho}{\longleftrightarrow} \frac{\omega x_{6}}{x_{2}}, \frac{x_{1}}{x_{6}} \stackrel{\rho}{\longleftrightarrow} \frac{\omega x_{58} x_{67}}{x_{13} x_{24}}, \\
\frac{x_{13}}{x_{58}} \stackrel{\rho}{\longleftrightarrow} \frac{x_{5}}{\omega}, \quad \frac{x_{24}}{x_{67}} \stackrel{\rho}{\longleftrightarrow} \frac{\omega}{x_{2}} .
\end{gathered}
$$


Then the symmetry properties of the weight function (15) are

$$
\begin{gathered}
R\left(u_{1}, u_{2}, u_{3}\right)_{i_{1} i_{2} i_{3}}^{j_{1} j_{2} j_{3}} \stackrel{\tau}{=} R\left(u_{3}, u_{2}, u_{1}\right)_{i_{3} i_{2} i_{1}}^{j_{3} j_{2} j_{1}}, \\
R\left(u_{1}, u_{2}, u_{3}\right)_{i_{1} i_{2} i_{3}}^{j_{1} j_{2} j_{3}} \stackrel{\rho}{=} R\left(\left(\omega u_{2}\right)^{-1}, u_{1},\left(\omega u_{3}\right)^{-1}\right)_{-j_{2} i_{1}-i_{3}}^{-i_{2} j_{1}-j_{3}} .
\end{gathered}
$$

When $q=1$, the above two relations reduce to the relations (44) and (45) in ref. [21]. From these properties we have

$$
R\left(u_{1}, u_{2}, u_{3}\right)_{i_{1} i_{2} i_{3}}^{j_{1} j_{2} j_{3}}=R\left(\left(\omega u_{3}\right)^{-1},\left(\omega u_{2}\right)^{-1}, u_{1}\right)_{-i_{3}-i_{2} j_{1}}^{-j_{3}} .
$$

It is a deformation of the star-star relation of the Baxter-Bazhanov model and reduces to the relation (39) of ref. [21] when $q=1$.

As the conclusions, we obtained the three-dimensional vertex model. The weight function of the model depends on four spins variables which are the linear combinations of the spins on the corner sites of the cube. The three spectrums of the vertex type weight function (15) relate the three 'space' of the function $R$ where a deformation parameter $q$ exists. The weight functions satisfy the modified vertex type tetrahedron equation where the six parameters are corresponding to six space on which the tetrahedron equation is defined. This vertex model is a duality of the BCC model. When $q=1$, the vertex model reduces to the one introduced in ref. [21]. The constraints are discussed also in detail. The symmetry properties of the weight function are given, from which we can get all of the symmetry transformations of the vertex type weight function under the action of the group $G$ consisting of the symmetrical maps of the three-dimensional cube. One of them is the deformation of the three-dimensional star-star relation of the Baxter-Bazhanov model.

Very recently, the modified tetrahedron equation was discussed also in ref. 225] and the vertex formulation of the Bazhanov-Baxter model is given, too, in ref. [26]. We know that this 3D lattice model was constructed firstly through the study of the chiral Potts model. And the chiral Potts model can be regarded as a descendant of the six-vertex model in two dimensions [2, 27]. The hidden symmetries of the six-vertex model transfer matrix and the correlation function [28, 29] of the $X X Z$ 
chain was discussed in ref. 30, 31] recently. What is the case in three dimensions? It is a interesting problem to discuss the correlation function and the free energy [32 for the three-dimensional integrable model. I hope the above discussions will be useful also for finding an algebra defined by $R L L L=L L L R$ similarly as the fusion of the Yang-Baxter equation in two dimensions.

\section{Acknowledgment}

The author would like to thank B. Y. Hou, K. J. Shi, Z. B. Su, P. Wang and K. $\mathrm{Wu}$ for the interesting discussions and J. Hietarinta, I. Korepanov and V. Korepin for the interests of this work. I am also grateful B. L. Hao for his reports at the meeting of statistical physics and nonlinear phenomena. 


\section{References}

[1] L. Onsager, Phys. Rev. 65 (1944) 117.

[2] E.H. Lieb, Phys. Rev. 162 (1967) 162; Phys. Rev. Lett. 18 (1967) 692, 1046; Phys. Rev. Lett. 19 (1967) 108.

[3] R.J. Baxter, Exactly Solved Models in Statistical Mechanics (Academic Press, London, 1982).

[4] R.J. Baxter, V.V. Bazhanov and J. H. H. Perk, Int. J. Mod. Phys. B 4 (1990) 803.

[5] A.B. Zamolodchikov, Commun. Math. Phys. 79 (1981) 489.

[6] V.V. Bazhanov and R.J. Baxter, J. Stat. Phys. 69 (1992) 453.

[7] R.M. Kashaev, V.V. Mangazeev and Yu.G. Stroganov, Int. J. Mod. Phys. A 8 (1993) 1399.

[8] Z.N. Hu and B.Y. Hou, Remarks on the Star-Triangle Relation in the BaxterBazhanov Model, J. Stat. Phys. (to appear).

[9] Z.N. Hu, Mod. Phys. Lett. B 8 (1994) 779.

[10] Z.N. Hu and B.Y. Hou, Cyclic Quantum Dilogarithm, Shift Operator and StarSquare Relation of BB Model, Phys. Lett. A (to appear).

[11] R.M. Kashaev, V.V. Mangazeev and Yu.G. Stroganov, Int. J. Mod. Phys. A 8 (1993) 587.

[12] Z. N. Hu, Int. J. Mod. Phys. 9 (1994) 5201.

[13] V.V. Bazhanov and R.J. Baxter, J. Stat. Phys. 71 (1993) 839.

[14] J. Hietarinta, J. Phys. A 27 (1994) 5727. 
[15] I. G. Korepanov, Commun. Math. Phys. 154 (1993) 85.

[16] C.-M. Viallet, Baxterization, Dynamical Systems, and the Symmetries of Integrability, preprint, PAR-LPTHE 94-08.

[17] J. Hietarinta, J. Phys. A 26 (1993) L9.

[18] M. Horibe and K. Shigemoto, On Solutions of Tetrahedron Equations Based on Korepanov Mechanism, preprint, November 1994.

[19] V.V. Mangazeev, S.M. Sergeev and Yu.G. Stroganov, New Solution of Vertex Type Tetrahedron Equations, hep-th/9410049, preprint (1994).

[20] Z.N. Hu, On the Three-dimensional Lattice Model, preprint (1995).

[21] Z.N. Hu and B.Y. Hou, Three-Dimensional Vertex Model in Statistical Mechanics, from Baxter-Bazhanov Model, preprint, hep-th/9503037.

[22] H.E. Boos, V.V. Mangazeev and S.M. Sergeev, Modified Tetrahedron Equation and Related 3D Integrable Models, preprint, hep-th/9407146

[23] V.V. Mangazeev, S.M. Sergeev and Yu.G. Stroganov, Int. J. Mod. Phys. A 9 (1994) 5517.

[24] V.V. Mangazeev and Yu.G. Stroganov, Mod. Phys. Lett. A 8 (1993) 3475.

[25] H.E. Boos, Modified Tetrahedron Equation and Related 3D Integrable Models, II, preprint, hep-th/9504119.

[26] S.M. Sergeev, V.V. Mangazeev and Yu.G. Stroganov, The Vertex Formulation of the Bazhanov-Baxter Model, preprint, hep-th/9504035.

[27] V.V. Bazhanov and Yu.G. Stroganov, J. Stat. Phys. 59 (1990) 799.

[28] M. Jimbo, K. Miki, T. Miwa and A. Nakayashiki, Phys. Lett. A 168 (1992) 256.

[29] N.S. Han and A. Honecker, J. Phys. A 27 (1994) 9. 
[30] I.G. Korepanov, Hidden Symmetries in the 6-Vertex Model of Statistical Physics, preprint, hep-th/9410066.

[31] F.H.L. Essler, H. Frahm, A.R. Its and V.E. Korepin, Integro-Difference Equation for a Correlation Function of the Spin- $\frac{1}{2}$ Heisenberg $X X Z$ Chain, preprint, BONN-TH-95-06.

[32] R.J. Baxter, Physica 18 D (1986) 321. 